\title{
Carfilzomib is an effective upfront treatment in AL amyloidosis patients with peripheral and
}

\section{autonomic neuropathy}

Richa Manwani ${ }^{1}$, Shameem Mahmood ${ }^{1,2}$, Sajitha Sachchithanantham ${ }^{1,2}$, Helen J Lachmann ${ }^{1}$, Julian D Gillmore ${ }^{1}$, Kwee Yong ${ }^{2}$, Neil Rabin ${ }^{2}$, Rakesh Popat ${ }^{2}$, Charalampia Kyriakou², Sarah Worthington ${ }^{2}$, Faye Sharpley ${ }^{1}$, Mark Smith ${ }^{2}$, Raakhee Shah ${ }^{2}$, Simon Cheesman ${ }^{2}$, Philip N Hawkins ${ }^{1}$ and Ashutosh D Wechalekar ${ }^{1,2^{*}}$

${ }^{1}$ National Amyloidosis Centre, University College London (Royal Free Campus), Rowland Hill Street, London

${ }^{2}$ Department of Haematology, University College London Hospitals, London, UK

* Corresponding Author:

Dr Ashutosh Wechalekar, National Amyloidosis Centre, University College London (Royal Free Campus), Rowland Hill Street, London, UK, NW3 2PF. Telephone number: +44 207433 2733, Fax number: +44 2074332817

Email: a.wechalekar@ucl.ac.uk

Text word count: 1293

Summary word count: 99

Number of figures: 1

Number of tables: 0

Number of references: 15 


\section{Summary}

Bortezomib is standard treatment in $\mathrm{AL}$ amyloidosis $(\mathrm{AL})$, but is contraindicated in patients with significant neuropathy. Carfilzomib, a second-generation proteosomal inhibitor, results in a lower incidence of neuropathy than bortezomib, but data in $A L$ is scant. We report a cohort of five AL patients treated with upfront carfilzomib. All had cardiac, peripheral and autonomic neuropathy at presentation. All achieved at least a very good partial haematological response. There was no worsening in cardiac function, peripheral or autonomic neuropathy. Carfilzomib is an effective upfront treatment option in AL patients with peripheral and/or autonomic neuropathy (without severe cardiac or renal involvement). 
Systemic AL amyloidosis ( $\mathrm{AL}$ ) is characterised by deposition of misfolded immunoglobulin light chains within organs. Treatment is based upon eradication of monoclonal amyloidogenic immunoglobulin light chain production with chemotherapy or autologous stem cell transplantation (ASCT). The latter, although associated with excellent haematological and organ responses, is unsuitable in most patients due to extent of amyloid-related organ dysfunction. Bortezomib, a proteasome inhibitor, has become the standard backbone of first-line therapy in ASCT-ineligible AL patients. Although neuropathy risk has been partially mitigated by subcutaneous, once-weekly administration, a third of patients treated with bortezomib develop peripheral neuropathy often necessitating its discontinuation.(Venner, et al 2012) Treatment of AL patients presenting with predominant amyloid-related peripheral or autonomic neuropathy (9-20\% of AL patients) remains challenging as bortezomib is contraindicated and alkylator-based regimes do not achieve the same depth of response.

Carfilzomib is a second-generation highly selective, irreversible proteasome inhibitor with well documented efficacy in newly diagnosed and relapsed myeloma, including in patients with bortezomib-resistant disease. Due to marked selectivity for the chemotrypsin-like active site of the proteasome(Parlati $F$ 2009), it has fewer off-target effects and in-vitro and animal studies have demonstrated its lesser neurotoxicity. (Arastu-Kapur, et al 2011, Kirk CJ 2008) Studies in myeloma have demonstrated a low incidence of treatment-emergent neuropathy with carfilzomib, with almost no zgrade 3 treatment-emergent peripheral neuropathy(Bringhen, et al 2014, Dytfeld, et al 2014, Hajek, et al 2017, Lendvai, et al 2014, Mikhael, et al 2015, Sonneveld, et al 2015) A Phase III study of carfilzomib-dexamethasone compared to bortezomib-dexamethasone demonstrated that the proportion of patients with grade $\geq 2$ peripheral neuropathy was significantly higher in the bortezomib group (32\%), compared to the carfilzomib group (6\%).(Dimopoulos, et al 2016) 
Carfilzomib is therefore potentially attractive in AL patients presenting with amyloid-related peripheral and/or autonomic neuropathy, but data on its use in AL is scant. In a phase I/II study of carfilzomib in 28 patients with relapsed/refractory AL, the maximal tolerated dose was $36 \mathrm{mg} / \mathrm{m}^{2}$ biweekly. (Cohen AD 2016) A quarter of patients had neuropathic disease. The overall response rate was $63 \%$, with $45.8 \%$ achieving a complete response (CR)/very good partial response (VGPR), but twice-weekly dosing was associated with substantial cardiac, pulmonary and renal toxicity. Worsening peripheral or autonomic neuropathy were not reported. A study of carfilzomib with thalidomide and dexamethasone in relapsed/refractory AL is ongoing at our centre, but excludes patients with significant neuropathy (ClinicalTrials.gov identifier NCT02545907). We report here a small cohort of AL patients with significant cardiac, peripheral nerve and autonomic involvement treated with carfilzomib at presentation.

Five patients with newly diagnosed AL who had cardiac, peripheral nerve and autonomic involvement were treated with carfilzomib at the UK National Amyloidosis Centre and University College London Hospitals. Bortezomib was contraindicated in all patients due to peripheral and autonomic nerve involvement. AL was confirmed on biopsy immunohistochemistry or proteomic analysis. All underwent serial biochemical tests for organ function, cardiac biomarkers, serum free light chains, serum and urine protein electrophoresis and immunofixation, echocardiography and cardiac MRI. Organ involvement was defined by international amyloidosis consensus criteria.(Gertz, et al 2005) Haematological responses were assessed according to AL response criteria.(Comenzo, et al 2012)

The median age was 62 years (40-69 years). All had cardiac involvement: Mayo (2004) Stage II and III (Dispenzieri, et al 2004)disease in 4/5 and $1 / 5$ patients, respectively. Two patients had ECOG score 1 and three had ECOG score 2. One patient and four patients was in NYHA class 1 and 2, respectively. The median NT-proBNP was 975ng/L (536-4681ng/L) and median serum troponin T was 43ng/L (37- 
90ng/L). Two patients had renal involvement. The median serum creatinine was 63umol/l (33$82 \mathrm{umol} / \mathrm{L})$, median eGFR $100 \mathrm{ml} / \mathrm{min}(86-100 \mathrm{ml} / \mathrm{min})$ and median 24 hour proteinuria was $0.2 \mathrm{~g} / 24$ hours (0.1-8.9g/24 hours). All had amyloid-related peripheral neuropathy and autonomic neuropathy. The median supine systolic blood pressure was $122 \mathrm{mmHg}(91-177 \mathrm{mmHg})$, with a median postural blood pressure fall of $27 \mathrm{mmHg}(20-44 \mathrm{mmHg})$. No patients had liver involvement; the median serum bilirubin was $4 \mu \mathrm{mol} / \mathrm{I}(2-12 \mu \mathrm{mol} / \mathrm{I})$ and median ALP was 74 units/L (62-110 units/L). The involved amyloidogenic light chain in all patients was lambda, with median presenting serum lambda light chains of $122 \mathrm{mg} / \mathrm{L}(64-208 \mathrm{mg} / \mathrm{L})$ and median difference in involved and uninvolved light chains (dFLC) of $97.8 \mathrm{mg} / \mathrm{L}(58.4-199.2 \mathrm{mg} / \mathrm{L})$.

All received weekly intravenous carfilzomib on days 1,8 and 15 (28 day cycle), combined with corticosteroids (intravenous methylprednisolone in three and dexamethasone in two patients). One patient each received additional lenalidomide or pomalidomide, respectively, for cycle 1 . The median number of cycles was 3 (range 1-6). The carfilzomib dose was $20 \mathrm{mg} / \mathrm{m}^{2}$ on Day 1 and $27 \mathrm{mg} / \mathrm{m}^{2}$ from Day 8 onwards. Two patients had dose escalation to $36 \mathrm{mg} / \mathrm{m}^{2}$ after cycle 1 in the absence of $\mathrm{CR}$ and one patient had a further dose increase to $56 \mathrm{mg} / \mathrm{m}^{2}$ from cycle 4 (achieving a CR after the first dose at this dose level).

Haematological responses were rapid with $80.6 \%$ (22-99.4\%) reduction in the median dFLC after one cycle. Figure 1 demonstrates dFLC values with each cycle. Three patients achieved a CR and two achieved a VGPR (one of the latter patients remains on carfilzomib-methylprednisolone). Both patients treated with an immunomodulatory agent achieved a CR at the end of cycle 1 . One patient achieved a cardiac response at 12 months, two did not, and two have not reached this assessment time-point. Of two patients with renal involvement, one did not achieve a renal response and the other has not reached the 12 month assessment time-point. 
In view of the small patient cohort in this cohort, Kaplan-Meier survival analysis was not performed.

One patient (in a CR) suffered a neck of femur fracture and died of post-operative complications. Although both patients treated with immunomodulatory combinations achieved a rapid CR, they discontinued treatment after one cycle due to drug-related problems: grade 3 fatigue and fluid retention with pomalidomide (this patient was severely nephrotic with serum albumin $<20 \mathrm{~g} / \mathrm{L}$ ) and a grade 3 rash with lenalidomide, respectively. The former patient progressed to requiring further treatment with an alternative regimen two months later. There have been no other progression events in the group.

Other grade 3 toxicity included transient acute kidney injury and hypotension in the patient treated with pomalidomide. Grade 1-2 toxicity included fatigue (4 patients), nausea (2 patients), diarrhoea (2 patients) and acute kidney injury (1 patient). All patients had an echocardiogram after cycle 1 and 2 with no deterioration in cardiac function. There was no worsening of peripheral or autonomic neuropathy.

This is the first report of carfilzomib in AL with significant neuropathy. Crucially, all patients had peripheral nerve, autonomic nerve and cardiac involvement at presentation and bortezomib was contraindicated due to extent of neuropathy. None experienced worsening peripheral or autonomic neuropathy with carfilzomib. None of the patients had any significant cardiovascular toxicity as assessed by echocardiography. Haematological responses were rapid and excellent, with all patients achieving a VGPR/better, and $3 / 5$ patients in a CR. The data is too limited to assess organ responses especially as follow-up is short and overall patient numbers small. This is a small, retrospective study with selected patients - none of whom presented with severely advanced cardiac involvement (all 
patients had an NT-proBNP<8500ng/L) or severe renal impairment. However, this study suggests that single agent carfilzomib has significant activity in AL. Whilst previous experience with carfilzomib in AL has demonstrated significant cardiac toxicity with a biweekly dosing regime(Cohen AD 2016), a once weekly protocol could be potentially administered without substantial cardiac toxicity and exacerbation of peripheral/autonomic neuropathy. Transient renal toxicity was noted in two patients, and close renal monitoring is critical in all AL patients treated with carfilzomib.

In conclusion, this data suggests that carfilzomib may be a potentially effective early treatment option in selected AL patients with peripheral and/or autonomic neuropathy (without severe cardiac or renal involvement) in whom bortezomib would be contraindicated. A prospective study in such patients is needed to confirm these findings. 
Figure 1: Serial dFLC (difference in involved and uninvolved light chains) values of patients in this cohort on carfilzomib treatment. 


\section{$\underline{\text { References }}$}

Arastu-Kapur, S., Anderl, J.L., Kraus, M., Parlati, F., Shenk, K.D., Lee, S.J., Muchamuel, T., Bennett, M.K., Driessen, C., Ball, A.J. \& Kirk, C.J. (2011) Nonproteasomal targets of the proteasome inhibitors bortezomib and carfilzomib: a link to clinical adverse events. Clinical Cancer Research, 17, 2734-2743.

Bringhen, S., Petrucci, M.T., Larocca, A., Conticello, C., Rossi, D., Magarotto, V., Musto, P., Boccadifuoco, L., Offidani, M., Omede, P., Gentilini, F., Ciccone, G., Benevolo, G., Genuardi, M., Montefusco, V., Oliva, S., Caravita, T., Tacchetti, P., Boccadoro, M., Sonneveld, P. \& Palumbo, A. (2014) Carfilzomib, cyclophosphamide, and dexamethasone in patients with newly diagnosed multiple myeloma: a multicenter, phase 2 study. Blood, 124, 63-69.

Cohen AD, L.H., Scott EC, Liedtke M, Kaufman JL, Rosenzweig M, Gasparetto C, Vesole DH, Sanchorawala V, Lentzsch S, Gomes CL, Comenzo RL, Durie BGM (2016) Safety and Efficacy of Carfilzomib (CFZ) in Previously-Treated Systemic Light-Chain (AL) Amyloidosis. Blood (ASH Annual Meeting Abstracts), 128, 645.

Comenzo, R.L., Reece, D., Palladini, G., Seldin, D., Sanchorawala, V., Landau, H., Falk, R., Wells, K., Solomon, A., Wechalekar, A., Zonder, J., Dispenzieri, A., Gertz, M., Streicher, H., Skinner, M., Kyle, R.A. \& Merlini, G. (2012) Consensus guidelines for the conduct and reporting of clinical trials in systemic light-chain amyloidosis. Leukemia, 26, 2317-2325.

Dimopoulos, M.A., Moreau, P., Palumbo, A., Joshua, D., Pour, L., Hajek, R., Facon, T., Ludwig, H., Oriol, A., Goldschmidt, H., Rosinol, L., Straub, J., Suvorov, A., Araujo, C., Rimashevskaya, E., Pika, T., Gaidano, G., Weisel, K., Goranova-Marinova, V., Schwarer, A., Minuk, L., Masszi, T., Karamanesht, I., Offidani, M., Hungria, V., Spencer, A., Orlowski, R.Z., Gillenwater, H.H., Mohamed, N., Feng, S. \& Chng, W.J. (2016) Carfilzomib and dexamethasone versus bortezomib and dexamethasone for patients with relapsed or refractory multiple myeloma (ENDEAVOR): a randomised, phase 3, open-label, multicentre study. Lancet Oncology, 17, 27-38.

Dispenzieri, A., Gertz, M.A., Kyle, R.A., Lacy, M.Q., Burritt, M.F., Therneau, T.M., Greipp, P.R., Witzig, T.E., Lust, J.A., Rajkumar, S.V., Fonseca, R., Zeldenrust, S.R., McGregor, C.G. \& Jaffe, A.S. (2004) Serum cardiac troponins and $\mathrm{N}$-terminal pro-brain natriuretic peptide: a staging system for primary systemic amyloidosis. Journal of Clinical Oncology, 22, 3751-3757.

Dytfeld, D., Jasielec, J., Griffith, K.A., Lebovic, D., Vesole, D.H., Jagannath, S., Al-Zoubi, A., Anderson, T., Detweiler-Short, K., Stockerl-Goldstein, K., Ahmed, A., Jobkar, T., Durecki, D.E., McDonnell, K., Mietzel, M., Couriel, D., Kaminski, M., Vij, R. \& Jakubowiak, A.J. (2014) Carfilzomib, lenalidomide, and low-dose dexamethasone in elderly patients with newly diagnosed multiple myeloma. Haematologica, 99, e162-164.

Gertz, M.A., Comenzo, R., Falk, R.H., Fermand, J.P., Hazenberg, B.P., Hawkins, P.N., Merlini, G., Moreau, P., Ronco, P., Sanchorawala, V., Sezer, O., Solomon, A. \& Grateau, G. (2005) Definition of organ involvement and treatment response in immunoglobulin light chain amyloidosis (AL): a consensus opinion from the 10th International Symposium on Amyloid and Amyloidosis, Tours, France, 18-22 April 2004. American Journal of Hematology, 79, 319328.

Hajek, R., Masszi, T., Petrucci, M.T., Palumbo, A., Rosinol, L., Nagler, A., Yong, K.L., Oriol, A., Minarik, J., Pour, L., Dimopoulos, M.A., Maisnar, V., Rossi, D., Kasparu, H., Van Droogenbroeck, J., Yehuda, D.B., Hardan, I., Jenner, M., Calbecka, M., David, M., de la Rubia, J., Drach, J., Gasztonyi, Z., Gornik, S., Leleu, X., Munder, M., Offidani, M., Zojer, N., Rajangam, K., Chang, Y.L., San-Miguel, J.F. \& Ludwig, H. (2017) A randomized phase III study of carfilzomib vs lowdose corticosteroids with optional cyclophosphamide in relapsed and refractory multiple myeloma (FOCUS). Leukemia, 31, 107-114.

Kirk CJ, J.J., Muchamuel T, et al. (2008) The selective proteasome inhibitor is well tolerated in experimental animals with dose intensive administration. Blood (ASH Annual Meeting Abstracts), 112, 11, Abstract 2765. 
Lendvai, N., Hilden, P., Devlin, S., Landau, H., Hassoun, H., Lesokhin, A.M., Tsakos, I., Redling, K., Koehne, G., Chung, D.J., Schaffer, W.L. \& Giralt, S.A. (2014) A phase 2 single-center study of carfilzomib $56 \mathrm{mg} / \mathrm{m} 2$ with or without low-dose dexamethasone in relapsed multiple myeloma. Blood, 124, 899-906.

Mikhael, J.R., Reeder, C.B., Libby, E.N., Costa, L.J., Bergsagel, P.L., Buadi, F., Mayo, A., Nagi Reddy, S.K., Gano, K., Dueck, A.C. \& Stewart, A.K. (2015) Phase Ib/II trial of CYKLONE (cyclophosphamide, carfilzomib, thalidomide and dexamethasone) for newly diagnosed myeloma. British Journal of Haematology, 169, 219-227.

Parlati F, L.S., Aujay M, et al. (2009) Carfilzomib: a selective inhibitor of the chymotrypsin-like activity of the constitutive proteasome and immunoproteasome has anti-tumor activity on multiple myeloma, lymphoma, and leukaemia cells with minimal effects on normal cells. Haematologica, 94, 0373.

Sonneveld, P., Asselbergs, E., Zweegman, S., van der Holt, B., Kersten, M.J., Vellenga, E., van Marwijk-Kooy, M., Broyl, A., de Weerdt, O., Lonergan, S., Palumbo, A. \& Lokhorst, H. (2015) Phase 2 study of carfilzomib, thalidomide, and dexamethasone as induction/consolidation therapy for newly diagnosed multiple myeloma. Blood, 125, 449-456.

Venner, C.P., Lane, T., Foard, D., Rannigan, L., Gibbs, S.D., Pinney, J.H., Whelan, C.J., Lachmann, H.J., Gillmore, J.D., Hawkins, P.N. \& Wechalekar, A.D. (2012) Cyclophosphamide, bortezomib, and dexamethasone therapy in $\mathrm{AL}$ amyloidosis is associated with high clonal response rates and prolonged progression-free survival. Blood, 119, 4387-4390.

\section{Acknowledgements}

We wish to thank those involved in the care of our patients, as well as our colleagues in histopathology, genetics, and nuclear and cardiac imaging.

\section{Conflict of interest disclosure}

None of the authors have conflicts of interest relevant to this manuscript.

\section{Authorship}

RM and ADW designed the study and wrote the manuscript. SM, SS, HJL, JDG, KY, NR, RP, CK, SW, FS, $\mathrm{MS}, \mathrm{RS}, \mathrm{SC}$ and PNH reviewed and critically appraised the manuscript. 